\title{
Integrating Functional and Structural Images for Simultaneous Cardiac Segmentation and Deformation Recovery ${ }^{\star}$
}

\author{
Ken C.L. Wong ${ }^{1}$, Linwei Wang ${ }^{1}$, Heye Zhang ${ }^{1}$, Huafeng Liu $^{2}$, \\ and Pengcheng $\mathrm{Shi}^{3,1}$ \\ ${ }^{1}$ Department of Electronic and Computer Engineering, \\ Hong Kong University of Science and Technology, Hong Kong \\ \{eewclken, maomwlw, eezhy, eeship\}@ust.hk \\ 2 State Key Laboratory of Modern Optical Instrumentation, \\ Zhejiang University, Hanzhou, China \\ liuhf@zju.edu.cn \\ ${ }^{3}$ School of Biomedical Engineering, Southern Medical University, Guangzhou, China
}

\begin{abstract}
Because of their physiological meaningfulness, cardiac physiome models have been used as constraints to recover patient information from medical images. Although the results are promising, the parameters of the physiome models are not patient-specific, and thus affect the clinical relevance of the recovered information especially in pathological cases. In view of this problem, we incorporate patient information from body surface potential maps in the physiome model to provide a more patient-specific while physiological plausible guidance, which is further coupled with patient measurements derived from structural images to recover the cardiac geometry and deformation simultaneously. Experiments have been conducted on synthetic data to show the benefits of the framework, and on real human data to show its practical potential.
\end{abstract}

\section{Introduction}

In order to describe the physiology of the heart, cardiac physiome models have been developed from invasive or in vitro experiments of anatomy, biomechanics, and electrophysiology. These models typically comprise an electrical propagation model (E model), an electromechanical coupling model (EM model), and a biomechanical model (BM model), which are connected together through a cardiac system dynamics. Because of their physiological meaningfulness, physiome models have been posed as model constraints to recover patient cardiac information from medical images. In the recently proposed cardiac kinematics recovery framework [1, given the image-derived motion information at salient features such as heart boundaries, the cardiac kinematics of the patient is recovered through the guidance of the physiome model with improved physiological

\footnotetext{
* This work is supported in part by the China National Basic Research Program (973-2003CB716100), and the Hong Kong Research Grants Council (CERGHKUST6151/03E).
} 
plausibility. The physiome model has also been used for recovering the local myocardial contractility from known displacement, with the aid of a relatively detailed anatomical heart model derived from patient's structural images [2]. Nevertheless, regardless of the promising potentials of the physiome models, most parameters used by these algorithms are not patient-specific, and thus the clinical relevance may be reduced especially under pathological conditions. Furthermore, following the spirit of [3], the segmentation and the deformation recovery tasks should be unified into a coherent process for more consistent and appropriate results.

In view of these problems, we propose a framework for simultaneous cardiac segmentation and deformation recovery, guided by a physiome model specified by information from body surface potential maps (BSPMs). Using the meshfree method [4, the heart is represented by a set of nodes bounded by surface elements, and simultaneous segmentation and deformation recovery is achieved by evolving the nodes through the physiome model and medical images. Adopting the recently proposed algorithm [5], the transmembrane potentials (TMPs) are recovered from BSPMs to give the patient-specific excitation sequence of the myocytes. This sequence is transformed into active contraction stresses through the EM model to provide the patient-specific physiological guidance for the recovery. On the other hand, a voxel matching algorithm based on comparing voxel similarities between two consecutive structural cardiac images is utilized to provide displacements of the boundary nodes as image-derived measurements. These BSPM-derived active stresses and image-derived measurements are coupled together through state-space equations composed of cardiac system dynamics, and filtering is applied to recover the cardiac geometry and deformation simultaneously in a statistically optimal sense. With this algorithm, functional images (BSPMs) are integrated with structural images through the physiome model to benefit both segmentation and deformation recovery. Experiments have been conducted on synthetic data to show the advantages of our framework, also on real human data to show its practical potentials.

\section{Methodology}

With the recent biological and technical breakthroughs, models describing cardiac physiology across different spatiotemporal scales are available, and appropriate models should be chosen depending on the specific purposes of the applications. As our proposed framework aims at recovering patient cardiac information from medical images, the cardiac physiome model being used should be able to capture the most significant cardiac physiological phenomena while ensure the computational feasibility of the complicated inverse problem. In consequence, we have chosen the FitzHugh-Nagumo model as the E model [5], a simple ordinary differential equation described in 2] as the EM model, and an anisotropic, elastic material model as the BM model [1]. These models are connected together through the total Lagrangian (TL) cardiac system dynamics to describe the relatively complete macroscopic physiology of the heart [1]. 
Using this physiome model as the central link, our proposed framework consists of three parts: integrating BSPMs with the physiome model to provide a relatively patient-specific physiological guidance, the voxel matching algorithm providing displacements of boundary nodes as image-derived measurements, and the state-space filtering framework coupling the measurements with the physiome model for simultaneous recovery of cardiac geometry and deformation.

\subsection{Integrating BSPMs with Physiome Model}

As described in the introduction, the physiome models used by the previous efforts are not patient-specific [12]. In order to partially address this problem, we utilize the algorithm proposed in [5] to recover TMPs from patient's BSPMs.

The algorithm consists of a spatiotemporal 3D TMP evolution model described via FitzHugh-Nagumo-like reaction-diffusion equations 1 :

$$
\left\{\begin{array}{l}
\frac{\partial \mathbf{U}_{e}}{\partial t}=-\mathbf{M}_{e}^{-1} \mathbf{K}_{e} \mathbf{U}_{e}+c_{1} \mathbf{U}_{e}\left(1-\mathbf{U}_{e}\right)\left(\mathbf{U}_{e}-a\right)-c_{2} \mathbf{U}_{e} \mathbf{V}_{e} \\
\frac{\partial \mathbf{V}_{e}}{\partial t}=b\left(\mathbf{U}_{e}-d \mathbf{V}_{e}\right)
\end{array}\right.
$$

where $\mathbf{U}_{e}$ and $\mathbf{V}_{e}$ are vectors of TMPs and recovery variables. $\mathbf{M}_{e}$ and $\mathbf{K}_{e}$, constructed based on the meshfree method, account for the intercellular coupling of electrical propagation. $a, b, c 1, c 2$ and $d$ are parameters defining the shapes of the action potentials.

To relate the observed BSPs with TMPs, the TMP-BSP projection model for the system-observation process follows the quasi-static electromagnetism:

$$
\boldsymbol{\Phi}=\mathbf{H}_{e} \mathbf{U}_{e}
$$

where $\boldsymbol{\Phi}$ represents BSPs and $\mathbf{H}_{e}$ is the transfer matrix obtained via a boundary element integral with embedded meshfree approximation.

Concerning about the system uncertainties of the TMP evolution model and observation errors of the measurements (BSPMs), (11) and (2) are written into state-space representations:

$$
\begin{aligned}
& \mathbf{x}_{e}\left(t+\Delta t_{e}\right)=F_{e}\left(\mathbf{x}_{e}(t)\right)+\omega_{e}\left(t+\Delta t_{e}\right) \\
& \mathbf{y}_{e}\left(t+\Delta t_{e}\right)=\tilde{\mathbf{H}}_{e} \mathbf{x}_{e}\left(t+\Delta t_{e}\right)+\nu_{e}\left(t+\Delta t_{e}\right)
\end{aligned}
$$

where $\mathbf{x}_{e}(\cdot)=\left[\mathbf{U}_{e}^{T}(\cdot) \mathbf{V}_{e}^{T}(\cdot)\right]^{T}$ is the state vector and $\mathbf{y}_{e}\left(t+\Delta t_{e}\right)=\mathbf{\Phi}\left(t+\Delta t_{e}\right)$ is the observation vector. $F_{e}(\cdot)$ is the transition function in correspondence with the E model (11) and $\tilde{\mathbf{H}}_{e}=\left[\mathbf{H}_{e} \mathbf{0}\right] \cdot \omega_{e}\left(t+\Delta t_{e}\right)$ and $\nu_{e}\left(t+\Delta t_{e}\right)$ are mutually independent vectors with zero-mean and known covariances, representing additive system uncertainties and observation errors.

With (3) and (4) available, unscented Kalman filtering described in [5] can be used to recover TMPs from patient BSPMs in a statistically optimal sense. Fig. 1 shows an example for the difference between TMPs obtained solely from the E model (Fig. 1(a)) and those recovered from BSPMs (Fig. 1(c)).

${ }^{1}$ In order to preserve the typical notations while avoiding confusion, some notations of the $\mathrm{E}$ model and the BM model are added with right subscripts $e$ and $m$ respectively. 

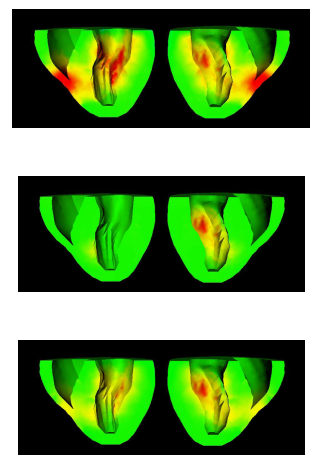
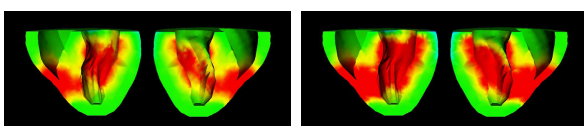

(a) Normal TMP propagation
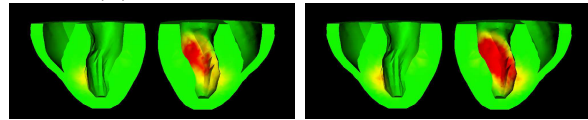

(b) RLPBBB TMP propagation
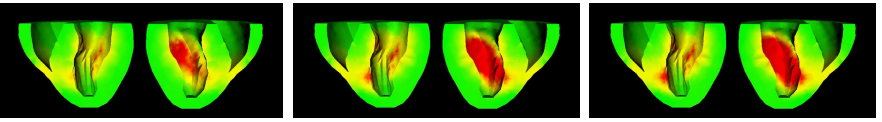

(c) RLPBBB TMP propagation recovered from BSPMs

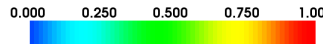

Fig. 1. Synthetic data. Volumetric TMP propagations at the beginning of ventricular excitations: depolarization at $1.5,2.7,5.4,7 \mathrm{~ms}$ (from left to right).

The recovered TMPs are then transformed into active contraction forces of the myocytes through the EM model [2], providing patient-specific physiological guidance to the recovery process through the cardiac system dynamics.

\subsection{Extracting Motion Measurements from Structural Images}

A voxel matching algorithm is used to provide displacements of the nodes representing the cardiac boundaries as the image-derived measurements.

Every boundary node is assumed to be lying on a voxel (source voxel) representing the heart boundary in the image at time $t$ (source image), and searching for a corresponding voxel (target voxel) in the image at time $t+\Delta t$ (target image). Every source voxel is given a searching window, within which the target voxel is the one which has the minimum value of:

$$
p=p_{\text {edginess }}\left[\alpha p_{\text {appearance }}+\beta p_{\text {shape }}\right]
$$

where $p_{\text {edginess }}$ is related to segmentation, $p_{\text {appearance }}$ and $p_{\text {shape }}$ are related to motion tracking. $\alpha$ and $\beta$ are selected to reflect the varying data constraints at different parts of the heart at different time frames.

As the new positions of the boundary nodes in the target image need to be on the voxels representing the heart boundaries for segmentation purpose, $p_{\text {edginess }}$ has the form:

$$
p_{\text {edginess }}=1 /(1+\|\nabla I\|)
$$

where $\|\nabla I\|$ is the magnitude of the image intensity gradient.

$p_{\text {appearance }}$ is the Gaussian weighted sum of the squared intensity difference between the voxel patches centered at the source voxel and the candidate target voxel respectively, thus it is related to the appearance similarity [6]. $p_{\text {shape }}$ is 

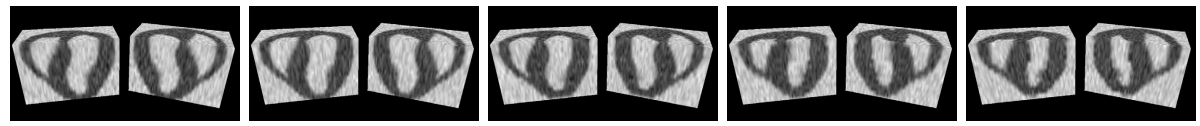

Fig. 2. Synthetic data. Structural images converted from synthetic heart deformations under RLPBBB condition (during systole, at 0, 60, 120, 180, and $240 \mathrm{~ms}$ ).

calculated in the same way as $p_{\text {appearance}}$, with the squared intensity difference replaced by the bending energy [7]:

$$
E_{\text {bending }}=0.5\left[\left(\kappa_{1, \text { source }}-\kappa_{1, \text { target }}\right)^{2}+\left(\kappa_{2, \text { source }}-\kappa_{2, \text { target }}\right)^{2}\right]
$$

where $\kappa_{1 \text {,source }}, \kappa_{1 \text {,target }}, \kappa_{2 \text {,source }}$, and $\kappa_{2 \text {,target }}$ are the principal curvatures of the isointensity surfaces in the source and target images [8]. Thus, $p_{\text {shape }}$ accounts for the shape coherence. As a result, the target voxel is lying on the heart boundary with similar appearance and/or shape to the source voxel.

The displacements of the boundary nodes become the image-derived measurements, providing patient's information for the recovery process.

\subsection{Simultaneous Cardiac Segmentation and Deformation Recovery}

In order to perform simultaneous cardiac segmentation and deformation recovery by evolving the node set representing the heart, state-space equations composed of the cardiac system dynamics is necessary to couple the BSPM-derived active stresses of section 2.1 with the image-derived measurements of section 2.2 . The cardiac system dynamics represented by the TL formulation is in the form [1]:

$$
{ }_{0}^{t} \mathbf{M}_{m}{ }^{t+\Delta t} \ddot{\mathbf{U}}_{m}+{ }_{0}^{t} \mathbf{C}_{m}{ }^{t+\Delta t} \dot{\mathbf{U}}_{m}+{ }_{0}^{t} \tilde{\mathbf{K}}_{m} \Delta \mathbf{U}_{m}={ }^{t+\Delta t} \mathbf{R}_{c}+{ }^{t+\Delta t} \mathbf{R}_{b}-{ }_{0}^{t} \mathbf{R}_{i}
$$

where ${ }_{0}^{t} \mathbf{M}_{m}$ and ${ }_{0}^{t} \mathbf{C}_{m}$ are the mass and damping matrices, and ${ }_{0}^{t} \tilde{\mathbf{K}}_{m}$ is the strain incremental stiffness matrix which contains the internal stresses, the material and deformation properties of the BM model at time $t .{ }^{t+\Delta t} \mathbf{R}_{c}$ is the force vector containing the BSPM-derived active stresses, ${ }^{t+\Delta t} \mathbf{R}_{b}$ is the force vector for enforcing boundary conditions, and ${ }_{0}^{t} \mathbf{R}_{i}$ is the force vector related to the internal stresses at time $t .{ }^{t+\Delta t} \ddot{\mathbf{U}}_{m},{ }^{t+\Delta t} \dot{\mathbf{U}}_{m}$ and $\Delta \mathbf{U}_{m}$ are the respective nodal acceleration, velocity and incremental displacement vectors at time $t+\Delta t$. The BSPM-derived active stresses is now connected with the cardiac kinematics through this cardiac system dynamics.

While the BSPM-derived active stresses provide a relatively patient-specific physiological guidance to the recovery process, however, most parameters of the physiome model still not coincide with the physiological properties of the patient, and thus introduce the system uncertainties. On the other hand, the cardiac images cannot provide perfect measurements of the patient, and thus introduce the observation errors. In order to compromise the patient measurements and the physiome model with each other, a statistical filtering framework is required 

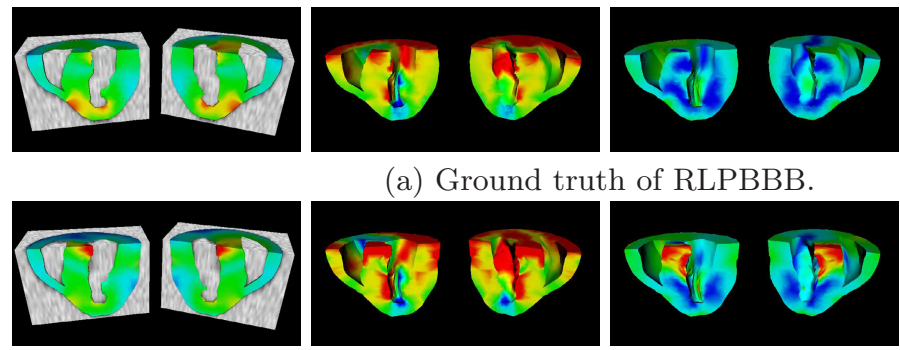

(a) Ground truth of RLPBBB.
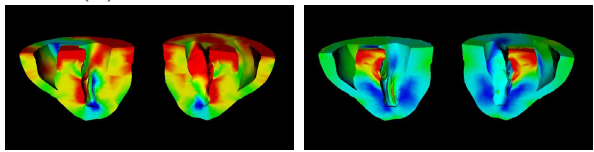

(b) Recovery without BSPMs information
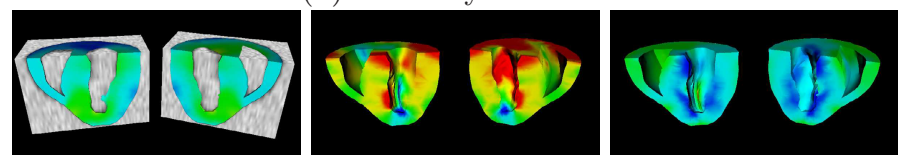

(c) Recovery with BSPMs information.

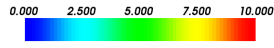

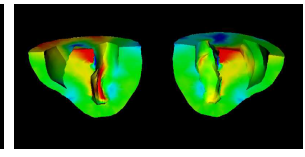
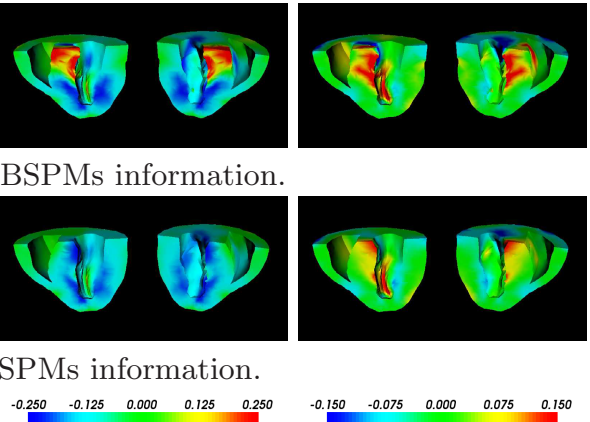

Fig. 3. Synthetic data. Geometry and kinematics at $180 \mathrm{~ms}$, defined on a cylindrical coordinate system $(r, \theta, z)$, with the long axis of the left ventricle as the $z$-axis. Left to right: displacement magnitude map overlapped with image, radial strain map, circumferential strain map and radial-circumferential strain map.

to couple them together. As a result, (8) is rewritten as a TL-updated state-space equation which performs nonlinear state (displacements) prediction [1]:

$$
\mathbf{x}_{m}\left(t+\Delta t_{m}\right)=\mathbf{F}_{m}(t) \mathbf{x}_{m}(t)+\mathbf{R}\left(t+\Delta t_{m}\right)+\omega_{m}\left(t+\Delta t_{m}\right)
$$

where $\mathbf{x}_{m}(t)={ }^{t} \mathbf{U}_{m}$ and $\mathbf{x}_{m}\left(t+\Delta t_{m}\right)={ }^{t+\Delta t_{m}} \mathbf{U}_{m}$ are the state vectors, containing the nodal displacements at time $t$ and $t+\Delta t_{m}$, the kinematics we want to recover. $\mathbf{F}_{m}(t)$ is the transition matrix comprised by the ${ }_{0}^{t} \mathbf{M}_{m},{ }_{0}^{t} \mathbf{C}_{m}$ and ${ }_{0}^{t} \tilde{\mathbf{K}}_{m}$ matrices. $\mathbf{R}\left(t+\Delta t_{m}\right)$ is the input vector which contains the right hand side of (8). $\omega_{m}\left(t+\Delta t_{m}\right)$ is the zero-mean, additive, and white system uncertainties.

Furthermore, as described in section 2.2 , the measurements are the imagederived, patient-specific displacements of the boundary nodes. In order to relate these measurements with the kinematics we want to recover, they are defined to be a subset of the state vector added by the zero-mean, additive, and white observation errors $\nu_{m}\left(t+\Delta t_{m}\right)$. Then the measurement vector $\mathbf{y}_{m}$ becomes:

$$
\mathbf{y}_{m}\left(t+\Delta t_{m}\right)=\mathbf{H}_{m} \mathbf{x}_{m}\left(t+\Delta t_{m}\right)+\nu_{m}\left(t+\Delta t_{m}\right)
$$

where $\mathbf{H}_{m}$ is the measurement matrix containing only 0 and 1 .

With the BSPM-derived active stresses ${ }^{t+\Delta t} \mathbf{R}_{c}$ embedded in (9) and the image-derived measurement vector $\mathbf{y}_{m}$ in (10), Kalman filtering procedures can be performed to evolve the whole node set representing the heart in a statistically optimal sense [1]. In consequence, the patient cardiac geometry and deformation are recovered simultaneously. 
Table 1. Synthetic data. Deviations of the recovered kinematics against the ground truth. Strains $\left(\epsilon_{\alpha \beta}\right)$ are calculated under a cylindrical coordinate system $(r, \theta, z)$, with the long axis of the left ventricle as the $z$-axis.

\begin{tabular}{ccc}
\hline \hline & Without BSPMs information & With BSPMs information \\
\hline $\mathbf{U}_{m}($ in $\mathrm{mm})$ & $0.9907 \pm 1.0800$ & $0.9760 \pm 1.0430$ \\
\hline$\epsilon_{r r}$ & $0.0341 \pm 0.0498$ & $0.0296 \pm 0.0434$ \\
\hline$\epsilon_{\theta \theta}$ & $0.0302 \pm 0.0501$ & $0.0272 \pm 0.0388$ \\
\hline$\epsilon_{z z}$ & $0.0447 \pm 0.4329$ & $0.0383 \pm 0.1741$ \\
\hline$\epsilon_{r \theta}$ & $0.0236 \pm 0.0373$ & $0.0221 \pm 0.0330$ \\
\hline$\epsilon_{\theta z}$ & $0.0212 \pm 0.0574$ & $0.0192 \pm 0.0282$ \\
\hline$\epsilon_{z r}$ & $0.0215 \pm 0.0550$ & $0.0193 \pm 0.0334$ \\
\hline \hline
\end{tabular}

\section{Experiments}

\subsection{Synthetic Data}

In order to show the benefits of our proposed framework, experiments have been conducted on synthetic data. Using the heart-torso model with geometry and cardiac fiber architecture adopted from 9] and 10, a specific pathological condition involving the right and left posterior fascicle branch bundle block (RLPBBB) has been studied. The major effect of the block is the disruption of the normal, coordinated and simultaneous distribution of the electrical signal to the two ventricles, which thus contract sequentially rather than simultaneously. The abnormal TMP propagation is shown against its normal counterpart in Fig. 1 (with 500ms in one cardiac cycle). The simulated BSPMs are mapped from the TMPs with 10dB SNR white Gaussian noises added. The cardiac deformations are also converted into a gray scale structural image sequence of 50 frames with image size $75 \times 75 \times 16$, added by $10 \mathrm{~dB}$ SNR white Gaussian noises so that the information provided are sparse and noisy (Fig. 2). These synthetic data are used as inputs to our experiments.

Cardiac geometry and kinematics are recovered using the physiome model without BSPMs information and our proposed framework. Fig. 1(c) shows the TMP propagation recovered from BSPMs, which is similar to the RLPBBB case and substantially different from the normal case. From the color maps shown in Fig. 3, it can be seen that the geometry and kinematics recovered using our proposed algorithm are closer to the ground truth. This is because complementary information from both BSPMs and structural images are utilized. The same conclusion can be made through the numerical results shown in Table 1 .

\subsection{Human Data}

Experiments on a normal human cardiac MR image sequence have been conducted to show that our proposed framework is applicable to real data with complicated structures. The image sequence contains 20 frames of a cardiac cycle. Each 3D image frame contains 8 image slices, with $10 \mathrm{~mm}$ inter-slice spacing, in-plane resolution of $1.56 \mathrm{~mm} /$ voxel, and temporal resolution of $43 \mathrm{~ms} /$ frame 

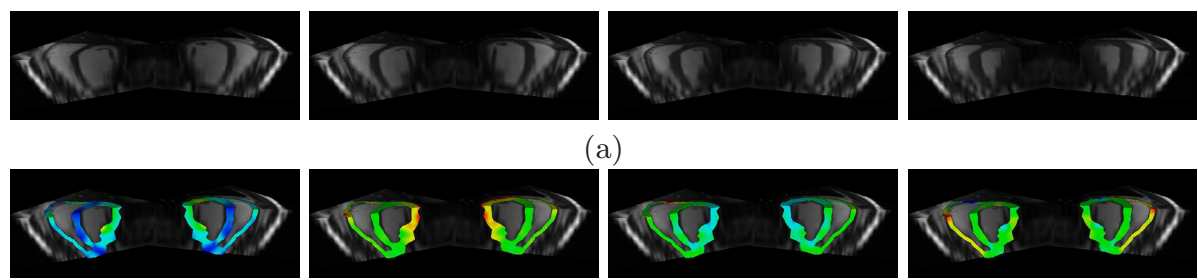

(a)
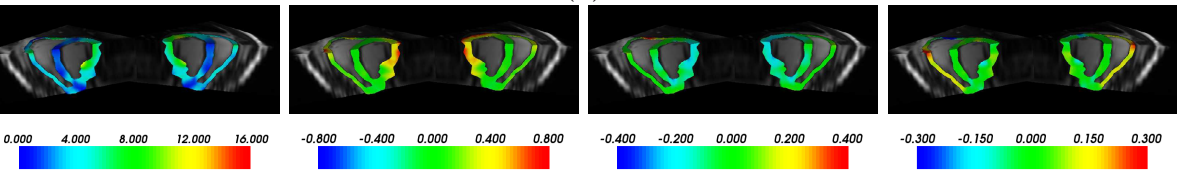

(b)

Fig. 4. (a). MR image sequence of a normal human heart during systole (frame \#1, \#3, $\# 5, \# 7$ ). (b). Recovered geometry and kinematics using the proposed algorithm (frame \#5). Left to right: displacement magnitude map, radial strain map, circumferential strain map, and radial-circumferential strain map.

(Fig. 4(a)). The initial meshfree representation of the heart is obtained by segmentation of the first image frame (end of diastole), and fibers are mapped from the fiber architecture of the heart model from the University of Auckland 110. Since the BSPMs of the patient are not available, synthetic BSPMs are currently used. Geometry and kinematics recovered using our proposed framework are shown in Fig. 4(b). Further experiments on diseased human and animal hearts are ongoing for further verifications.

\section{References}

1. Wong, K.C.L., Zhang, H., Liu, H., Shi, P.: Physiome model based state-space framework for cardiac kinematics recovery. In: Larsen, R., Nielsen, M., Sporring, J. (eds.) MICCAI 2006. LNCS, vol. 4190, pp. 720-727. Springer, Heidelberg (2006)

2. Sermesant, M., Moireau, P., Camara, O., Sainte-Marie, J., Andriantsimiavona, R., Cimrman, R., Hill, D.L.G., Chapelle, D., Razavi, R.: Cardiac function estimation from MRI using a heart model and data assimilation: Advances and difficulties. Medical Image Analysis 10(4), 642-656 (2006)

3. Paragios, N., Deriche, R.: Geodesic active contours and level sets for the detection and tracking of moving objects. IEEE TPAMI 22(3), 266-280 (2000)

4. Liu, G.: Meshfree Methods. CRC Press (2003)

5. Wang, L., Zhang, H., Shi, P., Liu, H.: Imaging of 3D cardiac electrical activity: a model-based recovery framework. In: Larsen, R., Nielsen, M., Sporring, J. (eds.) MICCAI 2006. LNCS, vol. 4190, pp. 792-799. Springer, Heidelberg (2006)

6. Anandan, P.: A computational framework and an algorithm for the measurement of visual motion. IJCV 2(3), 283-310 (1989)

7. Duncan, J., Lee, F., Smeulders, A., Zaret, B.: A bending energy model for measurement of cardiac shape deformity. IEEE TMI 10(3), 307-320 (1991)

8. Thirion, J., Gourdon, A.: Computing the differential characteristics of isointensity surface. Computer Vision and Image Understanding 61(2), 190-202 (1995)

9. MacLeod, R., Johnson, C., Ershler, P.: Construction of an inhomogeneous model of the human torso for use in computational electrocardiography. In: IEEE EMBS, pp. 688-689 (1991)

10. Nash, M.: Mechanics and material properties of the heart using an anatomically accurate mathematical model. PhD thesis, University of Auckland (1998) 